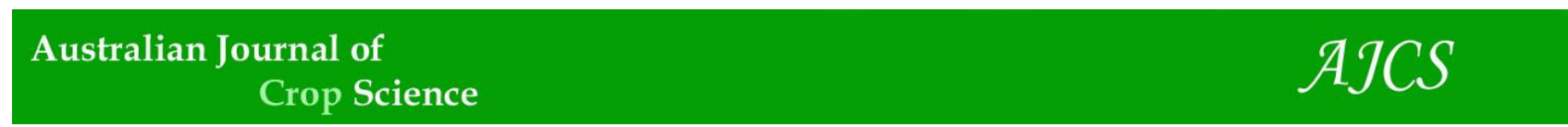

AJCS 11(04):406-418 (2017)

ISSN:1835-2707

doi: 10.21475/ajcs.17.11.04.pne290

\title{
Expression profile of gene encoding Kelch repeat containing F-box protein (PmF-box 1$)$ in relation to the production of green leaf volatiles
}

\author{
Muhamad Hafiz Che Othman ${ }^{1}$, Nurmahfuzah Abd Hadi ${ }^{1}$ Zamri Zainal ${ }^{1,2}$, Chew Jin Kiat ${ }^{2}$, \\ Muhammad Naeem-ul-Hassan ${ }^{1,3}$, Che Radziah Che Mohd. Zain ${ }^{1,2}$, Ismanizan Ismail ${ }^{1,2} *$
}

\author{
${ }^{1}$ School of Bioscience and Biotechnology, Universiti Kebangsaan, Malaysia \\ ${ }^{2}$ Institute of Systems Biology (INBIOSIS), Universiti Kebangsaan, Malaysia \\ ${ }^{3}$ Department of Chemistry, University of Sargodha, Pakistan
}

*Corresponding author: maniz@ukm.my

\begin{abstract}
Persicaria minor is one of the native aromatic plants in Malaysia and widely used in traditional foods and medicines. Previous studies have shown that treatment of $P$. minor with jasmonic acid upregulated genes involved in the secondary metabolites production and also gene encoding an F-box protein named PmFbox-1. It is known that F-box proteins play important roles in gene expression regulatory. However, the function of PmF-box 1 protein is still unknown. In this study, the full length cDNA sequence of PmF-boxl, with NCBI accession number JQ429325, consisting of 2768 bp was isolated followed by characterization of the deduced protein sequence of 487 amino acids. The putative F-box protein belongs to Kelch repeat-containing F-box protein family (KFB) and has a moderate level of homology with other F-box proteins from different organisms, showing a maximum identity score of $60 \%$ with an F-box motif and two conserved kelch repeat motifs. Time course semi-quantitative reverse-transcriptase PCR gel image analyses showed that $P m F$-boxl was induced after JA treatment, indicating that $P m F$-boxl is a JA inducible gene. $P m F$-boxl also differentially expressed in different tissues after treatment with JA, indicating a spatial regulation of its activity. Interestingly, there was high correlation in the expression pattern between PmF-boxl and PmADH1 (P. minor Alcohol dehydrogenase 1$)(\mathrm{R}=0.90 \pm$ 0.05) after treatment with $150 \mu \mathrm{M} \mathrm{JA}$, indicating that PmF-box 1 possibly involves in regulating the activity of PmADH1. The content of hexanal was significantly increased in JA-treated plants and even higher in JA plus SA-treated plants. The level of six carbon alcohols was increased at 48 hours after JA treatment, consistent with the upregulation of PmADHI in JA treated plants. These results suggest that PmF-boxl encodes a KFB-type F-box protein that may be involved in plant stress signaling by influencing the expression of $P m A D H 1$; thus, modulating the production of green leaf volatiles.
\end{abstract}

Received 13 Aug 2016; Revised 29 Nov 2016; Accepted 13 Feb 2017.

Keywords: Kelch repeat containing F-box proteins (KFB); Plant stress responses; Alcohol dehydrogenase (ADH); Lipoxygenase (LOX); Polygonum minus H.; Green leaf volatiles (GLVs).

\section{Introduction}

Under the given set of any environmental conditions, plants continuously perceive stresses that may be either biotic or abiotic in nature. Over the course of time, plants are adapted to these conditions and defend themselves from these stresses through the activation of various mechanisms, leading to expression of stress responsive genes and other physiological changes. In planta, induction of the defence responses is largely mediated by the production of signalling molecules such as JA, SA, and ethylene (Verhage et al. 2010). Furthermore, exogenous application of these signalling molecules to the plant surface can also induce the defence responses in the plant (Bari and Jones 2009). Numerous signal transduction components of plants, which play crucial roles in transferring the stress signals to the nucleus have been studied using the model plant Arabidopsis thaliana. One of the major signal transduction component, involved in almost every aspect of plant growth and development is mediated through the activities of F-box proteins.

F-box proteins constitute the most important subunit of ubiquitin-protein ligase (EC 6.3.2.19) complex known as SCF complex (which stands for its SKP1/ ASK, Cullin and F-box subunits). This complex serves as an ubiquitin ligase in the degradation of regulatory proteins by ubiquitin proteasome system (UPS) (Hua and Vierstra 2011). The ubiquitin is first activated by the E1 enzyme and then transferred to the E2 enzyme. The SCF complex, which is one of the most common E3 enzyme, transfers the ubiquitin chains from E2 to the target proteins before its modification or degradation by $26 \mathrm{~S}$ proteasome (Lechner et al. 2006). F-box proteins play a crucial role in the SCF complex as it confers specificity to the complex toward the target proteins. F-box proteins are characterized by containing an F-box domain of about 40-50 amino acids on the $\mathrm{N}$-terminal. The C-terminal of F-box proteins is comprised of variable number of different types of protein-protein interaction domains, such as WD-40 repeats, leucine rich repeats (LRR), kelch repeats and other motifs (Ho et al. 2008). F-box proteins interact with the SKP1/ASK through the F-box domain and with the target protein through the downstream protein-protein interaction domain. Arabidopsis has been reported to contain more than 700 F-box proteins and thought to be involved in 
modulating various cellular processes including plant hormone signalling, circadian oscilation, flower formation and stress responses (Somers and Fujiwara 2009).

The roles of F-box proteins in stress signalling of the jasmonate (JA) signalling pathway have been very well studied (Paquis et al. 2010; Somers and Fujiwara 2009; Thines et al. 2007). CORONATINE-INSENSITIVE 1 (COI1), the only Fbox protein known to be involved in the JA signalling, will bind to jasmonyl-isoleucine when the JA levels are increased due to stress (Heil and Ton 2008). COI1 belongs to LRR containing Fbox proteins. The binding of jasmonyl-isoleucine to COI1 subunit of $\mathrm{SCF}^{\mathrm{COI} 1}$ complex promotes the interaction between the COI1 subunit to its target protein, the JAZ (jasmonate ZIMdomain) proteins. This interaction directs the repeated ubiquitination of JAZ proteins followed by their rapid degradation through the UPS. JAZ proteins serve as repressors for the expression of JA-inducible genes. Their degradation allows the transcription factors to stimulate the expression of JA-inducible genes and initiate the JA related defence responses (Thines et al. 2007).

One of the major components in the JA-related defence responses is the production of green leaf volatiles (GLV), which occurs especially in response to herbivory, wounding and pathogen attack (Piesik et al. 2011; Wenda-Piesik et al. 2010). GLV consist of short chain aldehydes, alcohols and their esters derived by the sequential action of lipoxygenase (LOX, EC 1.13.11.12), hydroperoxide lyase (HPL, E.C. 4.1.2.) and downstream enzymes like alcohol dehydrogenase (ADH, EC 1.1.1.1) of the oxylipin pathway (Gounaris 2010). In Arabidopsis, a LOX enzyme that is encoded by a JA induced gene, catalyzes the peroxidation of specific double bonds of unsaturated fatty acids, mainly linoleic acid and $\alpha$-linolenic acid to produce the corresponding hydroperoxides (Combet et al. 2006). These hydroperoxides are then converted to various volatile compounds, consisting of $\mathrm{C}_{6}$ and $\mathrm{C}_{9}$ aldehydes, by HPL (Wang et al. 2001). ADH, also encoded by a JA induced gene in Arabidopsis, plays its role in interconversion of the volatile compounds between the aldehyde and the alcohol forms (Longhurst et al. 1990). Previous studies showed that suppression of LOX genes in tomato (Lycopersicon esculentum) and potato (Solanum tuberosum) reduced the production of GLV (Chen et al. 2004; León et al. 2002), while overexpression of the soybean $L O X$ in tobacco (Nicotiana tabacum) increased $\mathrm{C}_{6}$ aldehyde production (Deng et al. 1992). Over expression of $\mathrm{ADH}$ genes has been shown to increase $\mathrm{C}_{6}$ alcohols in ripe tomato fruits (Speirs et al. 1998) and both $\mathrm{C}_{6}$ alcohols and aldehydes in grape (Tesniere et al. 2006).

A previous study in our lab has revealed that in Persicaria minor, a gene encoding a kelch repeat containing F-box protein named PmF-boxl, an ADH gene named PmADHI and a LOX gene named $P m L O X$ were found differentially expressed in $P$. minor after the plants were treated with JA (Gor et al. 2010). $P$. minus or commonly known as kesum is very famous among Malaysians as a plant that exerts sweet and pleasant aroma. It also has been acknowledged by the Malaysian government in the Herbal Product Blueprint as an essential oil-producing crop (Wan Hassan 2007). With these characteristics kesum is very suitable to be used to study the possible relationship between the role of PmF-box 1 and the production of GLVs through JA signaling pathway. Until now, there is no any kelch repeat containing F-box protein that has been studied in relation to JA signalling. Moreover, not even a single F-box protein has been explored for its function in the production of GLV. Therefore, present study was designed to investigate the structure and function of PmF-boxl in more detail and to evaluate its possible involvement in the GLV production. Hence, the first objective of the present study was to obtain the full length sequence of PmF-boxl cDNA. The second aim was to analyse the expression pattern of the gene after different hormone treatments in various tissues and determine its influence on the GLV production through activities of $P m A D H 1$ and $P m L O X$.

\section{Results}

Isolation and characterization of the sequence of PmF-boxI gene

Two fragments of around $1900 \mathrm{bp}$ and $900 \mathrm{bp}$ sizes were produced by $5^{\prime}$ and $3^{\prime}$ RACE respectively. After plasmid construction followed by transformation and cDNA clone sequencing and by removing the vector and adapter sequences, $1975 \mathrm{bp}$ sequence was obtained by 5' RACE and $916 \mathrm{bp}$ fragment for the $3^{\prime}$ RACE. The full-length cDNA sequence of $2768 \mathrm{bp}$ was obtained by aligning and assembling the $5^{\prime}$ and $3^{\prime}$ RACE fragments.

Verification of the sequence was accomplished by PCR using a pair of primers to amplify the full length sequence (the sense primer located before the start codon ATG, and the reverse primer located after the stop codon TAA) (Table 1). Total RNA extracted from the kesum leaves was used to prepare the first strand cDNA that was used as template in the PCR. The resulting amplicon suited the expected size, which was further verified by sequencing. The sequence analysis indicated that the cDNA sequence of PmF-boxl consists of an open reading frame (ORF) of $1464 \mathrm{bp}$ flanked by 5 '- and 3 '-untranslated regions of $616 \mathrm{bp}$ and $688 \mathrm{bp}$ respectively. The deduced PmFbox 1 protein has 487 residues with a calculated molecular mass of 53,359 Da. The sequence of PmF-boxl was deposited in genetic sequence database at the National Center for Biotechnical Information (NCBI), and was assigned a GenBank ID JQ429325. The primer pair used for RACE validation was used to amplify the PmF-boxl from P. minor genome. The sequencing results indicated that, corresponding to its cDNA sequence, the amplified genomic PmF-boxl sequence that has the size of $2776 \mathrm{bp}$ contains one intron. This intron is located within the 5 ' untranslated region of the gene, and the length of the intron has been found $711 \mathrm{bp}$. As the primers used to amplify genomic PmF-boxl sequence were designed based on the sequence laying within the UTR sequences, therefore some of the nucleotide sequence would not be amplified. By adding up the nucleotide sequence of PmF-boxl which was not amplified by the abovementioned primer set, the actual size of the genomic sequence of PmF-boxl should be $3479 \mathrm{bp}$. Schematic diagram for obtaining full length cDNA sequence of PmF-boxl is shown in Fig 1.

\section{Phylogenetic analysis and domain organization of PmF-boxl putative protein}

BLASTp search shows that PmF-box 1 protein was moderately identical to other homologous F-box proteins, with highest identity score of $60 \%$ within the dicotyledonous branch and $58 \%$ within the monocotyledonous plants. Eleven protein sequences from different plant species that have highest sequence similarity with PmF-box1 were selected for phylogenetic analysis. The phylogenetic analysis divided these F-box proteins into monocotyledon and dicotyledonous clades (Fig 2). PmF-box1 belongs to the dicotyledonous clade and further placed within the same subclade as for the Arabidopsis 
Table 1. Sequences of primers used for RACE and Race validation.

\begin{tabular}{ll}
\hline Purpose & Primer sequences \\
\hline RACE & 5' end GSP: 5'-CAATGACTCCTTCACCATAAGCCCTG-3' \\
RACE validation & 3' end GSP: 5'-TCCACTGTTGGGAAACTGCCTGA-3' \\
& F: 5'- TTTTGCTCATTCGTGGCTCT-3' \\
& R: 5' - TAACTGAGTTATGCGACGCC-3' \\
\hline
\end{tabular}

Table 2. Reference proteins that were used in I-Tasser in the prediction of PmF-box 1 protein structure.

\begin{tabular}{lll}
\hline PDB ID & Protein name & Normalised Z-score \\
\hline 2xn4A & Kelch domain of human KLHL2 & 2.40 \\
1gofA & Galactose oxidase & 2.81 \\
2wozA & B-propeller of the btb-kelch protein KRP1 & 4.17 \\
1k3iA & Precursor of galactose oxidase & 2.45 \\
3ii7A & Kelch domain of human KLHL7 & 3.86 \\
1u6dX & Kelch domain of human Keap1 & 5.09 \\
\hline
\end{tabular}

PDB ID: Protein Data Bank Identification; Normalised Z-score: Normalised Z-score of the threading alignments. Alignment with a Normalised Z-score $>1$ mean a good alignment and vice versa.

Table 3. Sequences of primers used for semi-quantitative RT-PCR.

\begin{tabular}{ll}
\hline Gene & Primer sequences \\
\hline PmF-box1 & F: 5'-TCCACTGTTGGGAAACTGCCTGA-3' \\
& R: 5'-CAATGACTCCTTCACCATAAGCCCTG-3' \\
PmLOX & F: 5'-TCTTCATAAACTCCCACCCAAAATCC-3' \\
& R: 5'-TAAGGAGTTGGAGGGGAAGAGGTTT-3' \\
PmADH1 & F: 5'-TGGTTCCTGGTAAAAGCCGTTGC-3' \\
& R: 5'-CCACGGATTATTCCATTGACCCTG-3' \\
PmCyp & F: 5'-CCAAAGACAGCAGAAAACTTCCGTG -3' \\
& R: 5'- TCAGGTGGGTGAGATAAAACAGACG -3' \\
\hline
\end{tabular}

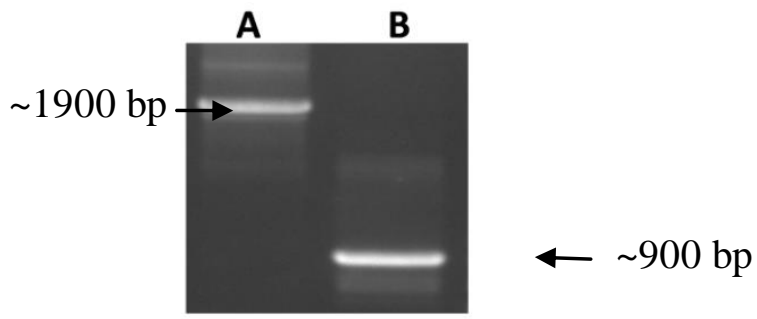

(I)
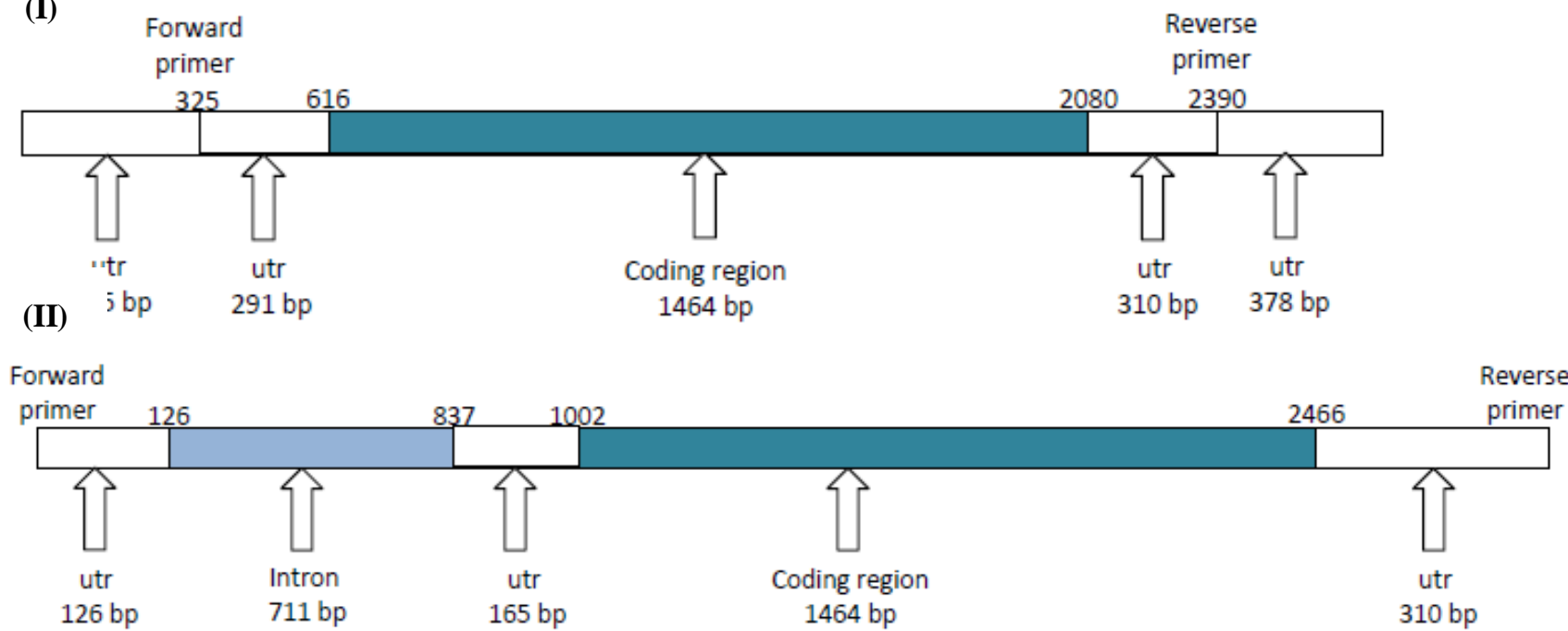

Fig 1. Obtaining the full length cDNA and genomic sequence of PmF-box 1 through RACE-PCR. A and $\mathbf{B}$ are the 5' and 3' RACE products for the isolation of full length cDNA of PmF-box 1 respectively. (I) The structure of full length cDNA of PmF-box 1, (II) the structure of amplified genomic fragment. 


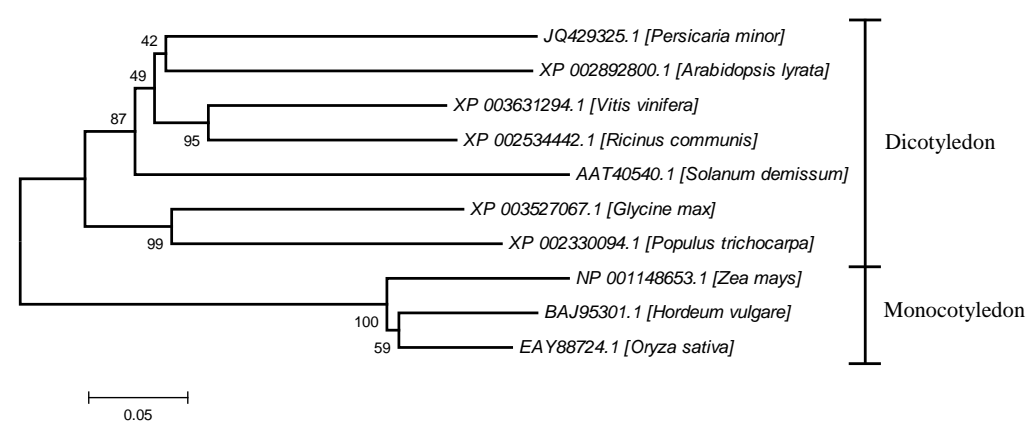

Fig 2. Phylogenetic relationship of PmF-box1 (AFD02178.1) with homologous F-box amino acid sequences from Solanum demissum (AAT40540.1), Arabidopsis lyrata (XP002892800.1), Arabidopsis thaliana (NP178390.1), Vitis vinifera (XP003631294.1), Ricinus communis (XP002534442.1), Glycine max (XP003527067.1), Populus trichocarpa (XP002330094.1), Hordeum vulgare (BAJ95301.1), and Zea mays (NP_001148653.1) and Oryza sativa Kumpulan Indika (EAY88724.1). The neighbour-joining method was used to construct the tree. Numbers on nodes indicate the bootstrap values after 1,000 replicates.
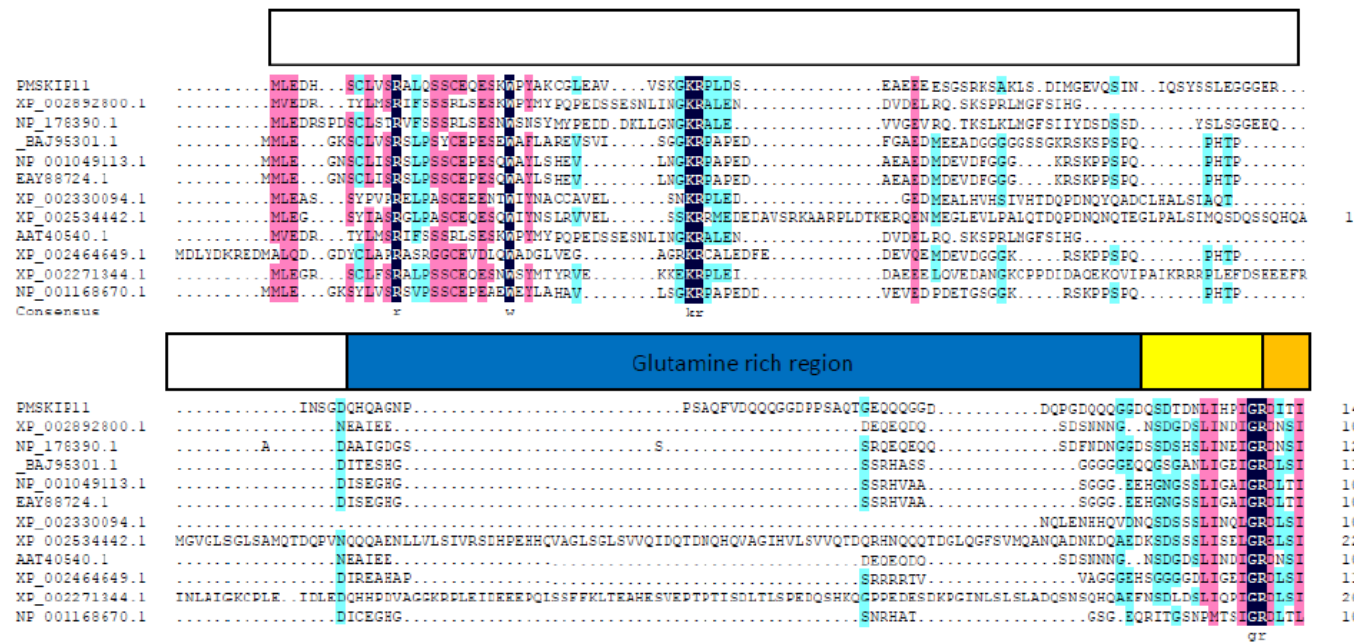

$$
\begin{aligned}
& 149 \\
& 104 \\
& 127 \\
& 113 \\
& 105 \\
& 105 \\
& 104 \\
& 220 \\
& 104 \\
& 118 \\
& 203 \\
& 103
\end{aligned}
$$
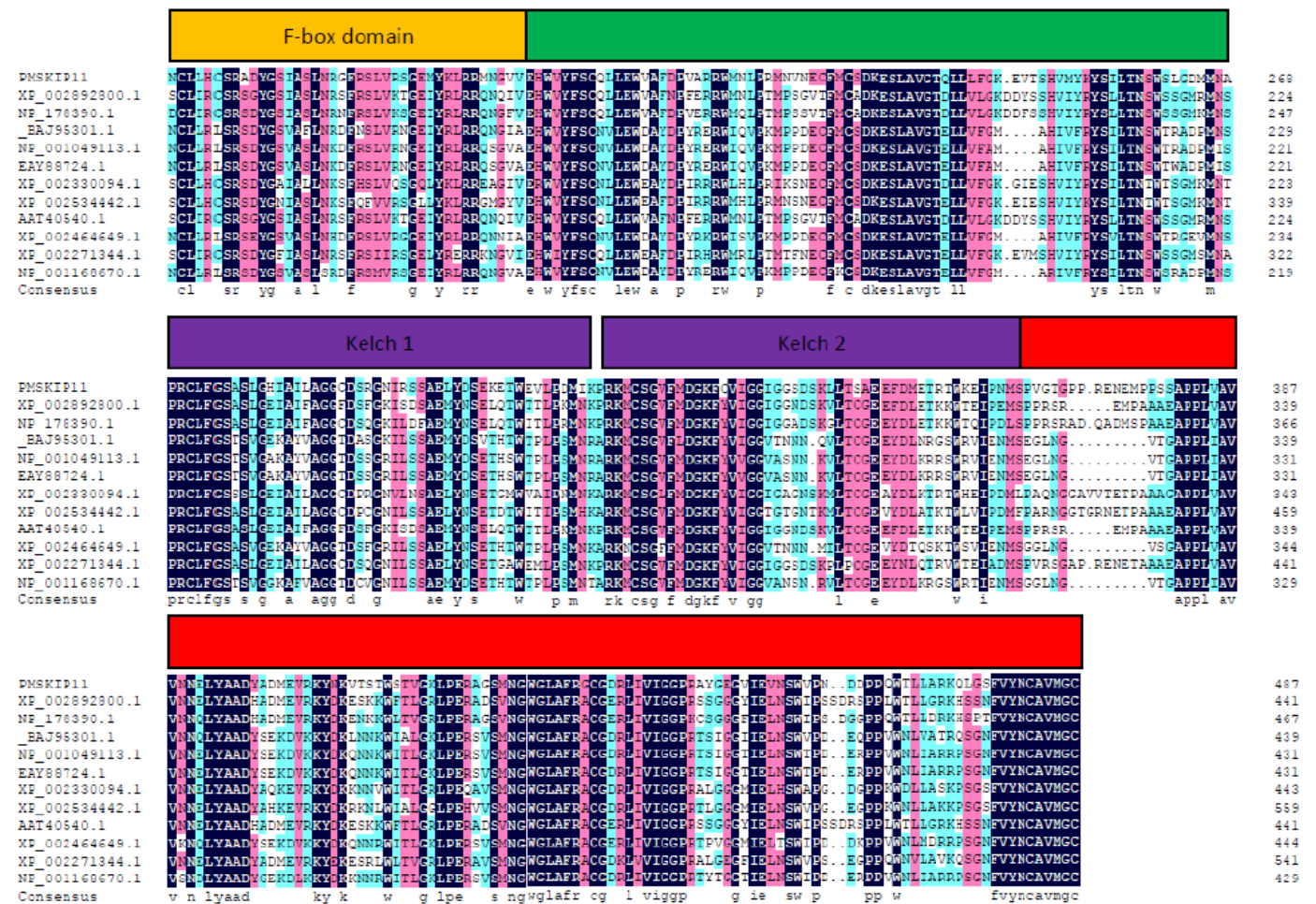

Fig 3. Alignment of the putative amino acid sequences of PmF-box 1 with homologous F-box sequences from other plant species. The specific sites for F-box, and kelch repeat motifs have been indicated. 
a)

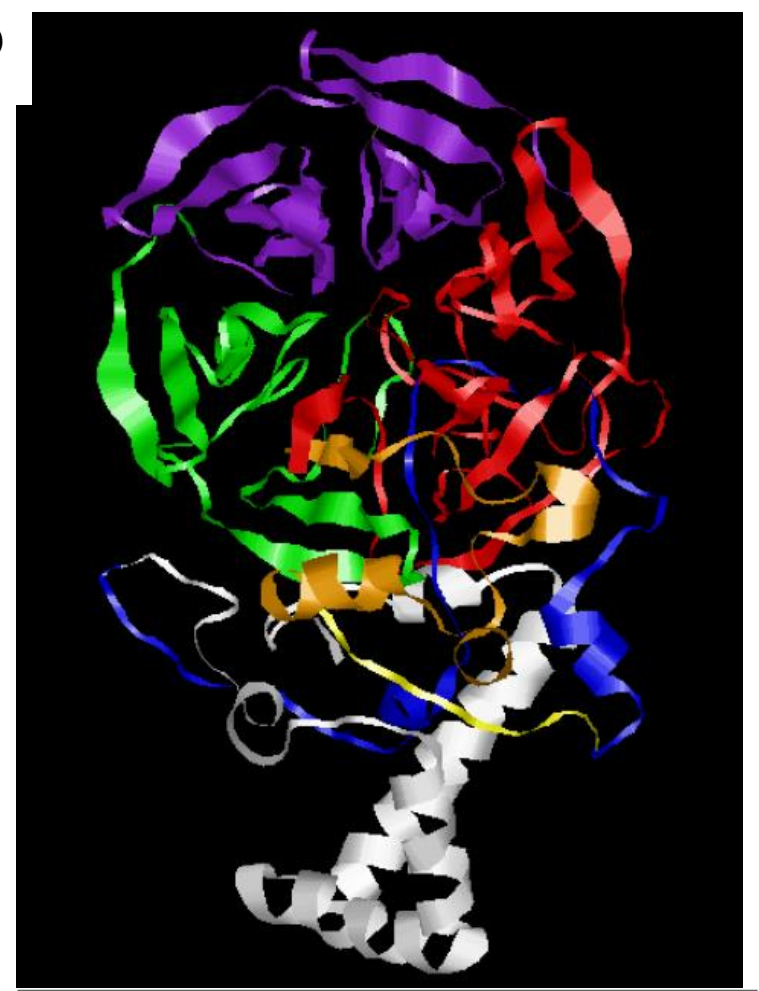

c)

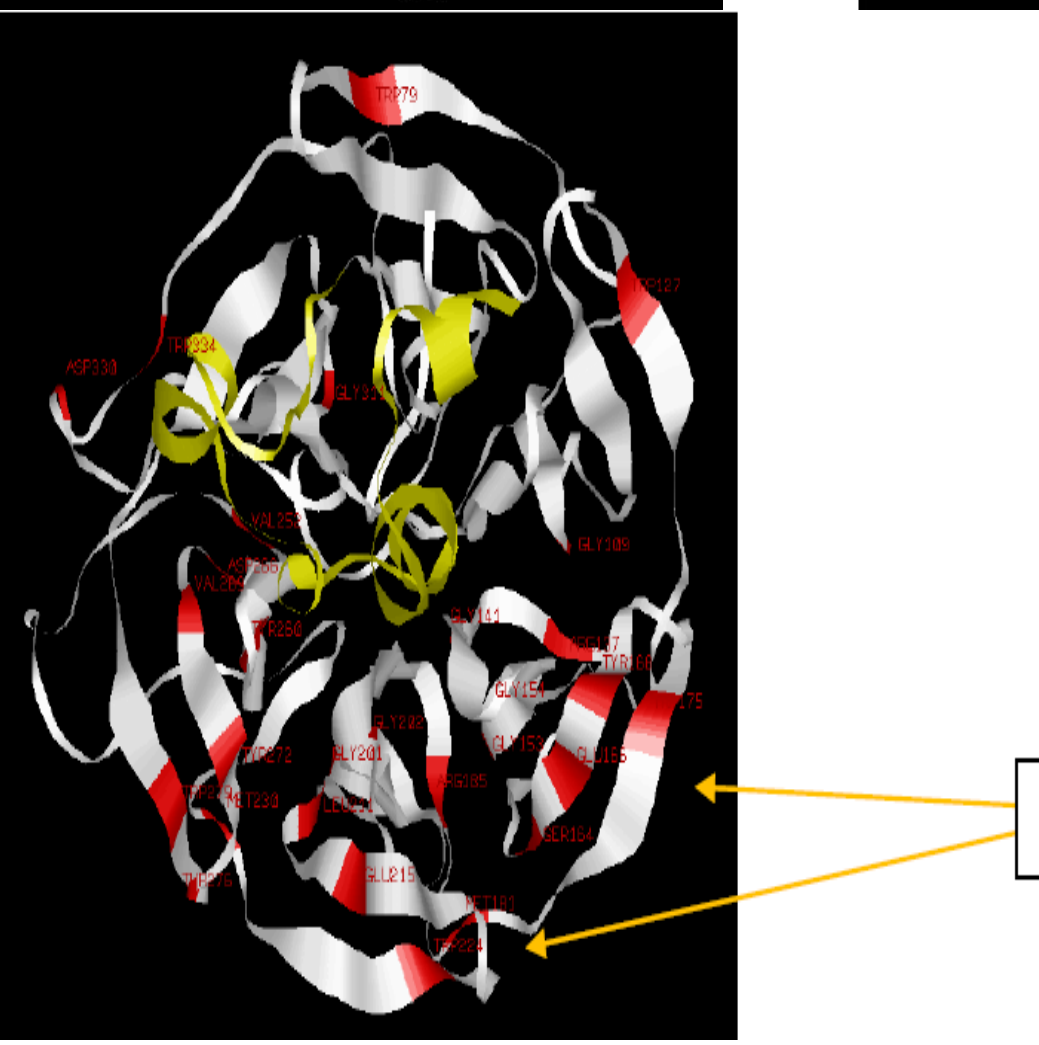

b)

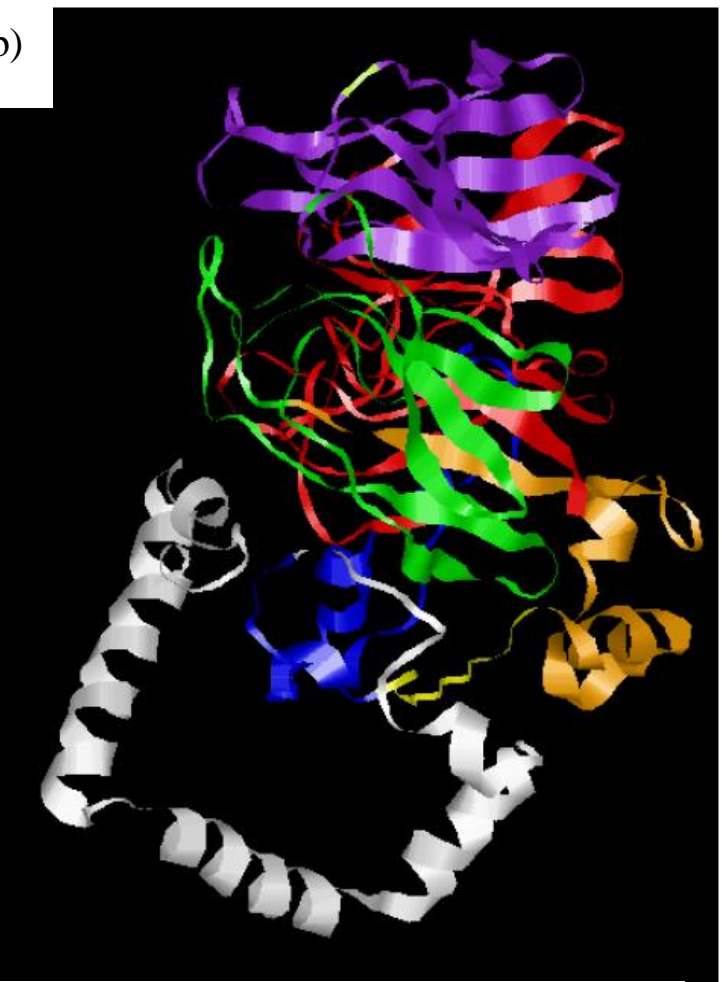

\section{Conserved Kelch repeat domains}

Fig 4. Tertiary protein structure of PmF-box 1 predicted by the online protein structure predictor tool I-Tasser. a) Front view of the predicted tertiary structure. b) Side view of the predicted tertiary structure. c) Close-up view on the C-terminal domain of predicted tertiary structure of PmF-box1. The conserved kelch repeat domains identified through motif scan were indicated by arrows. 


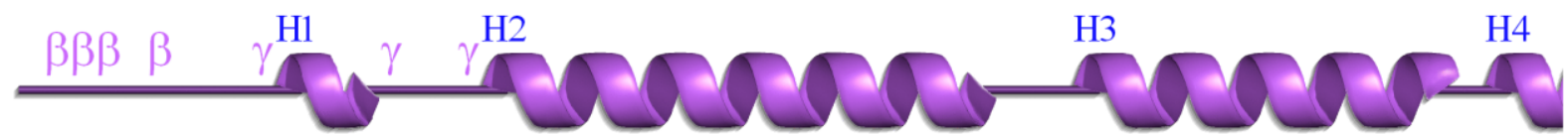

MLEDHSCLVSRALQS SCEQESKWPYAKCGLEAVVSKGKRPLDSEAEEEE SGSRKSAKL SD

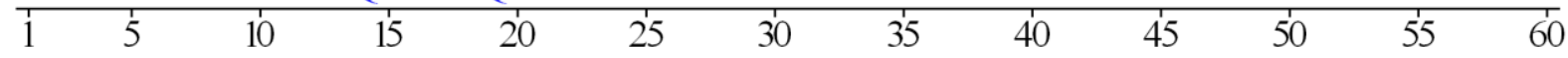

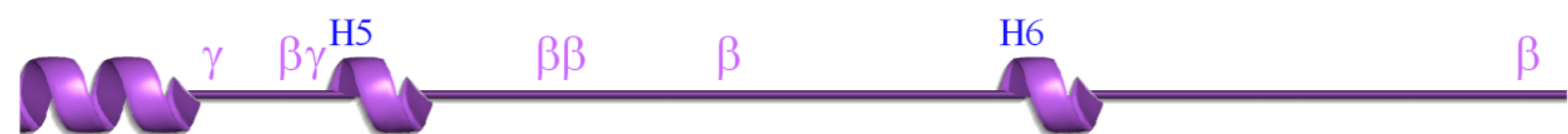

IMGEVQS IN IQSYS SLEGGGER I NSGDQHQAGNP P S AQFVDQQQGGDP P SAQTGEQQQGG

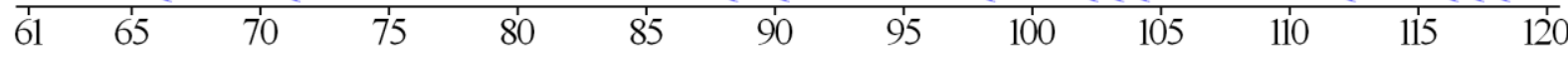

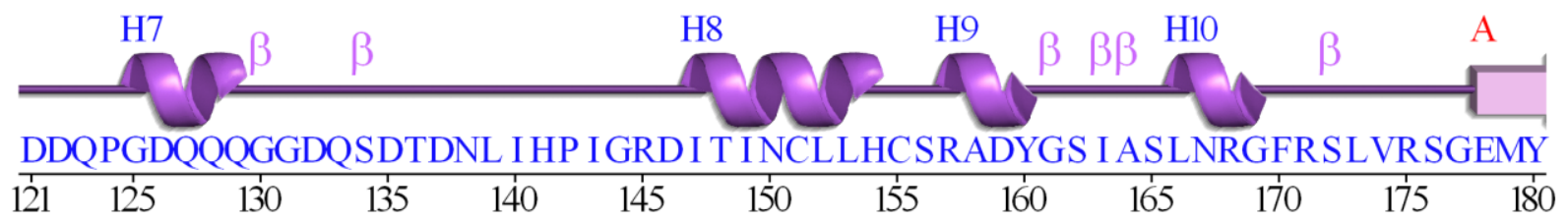

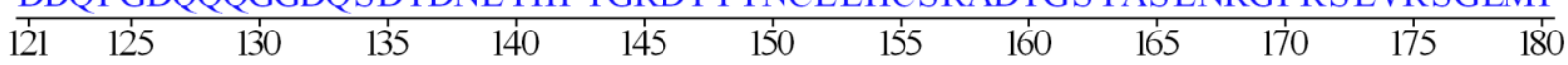

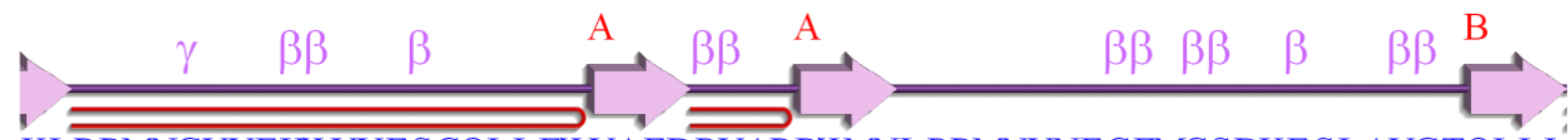

KLRRMNGVVEHWVYF SCQLLEWVAFDPVARRWMNL PRMNVNECFMC SDKESLAVGTQLLL

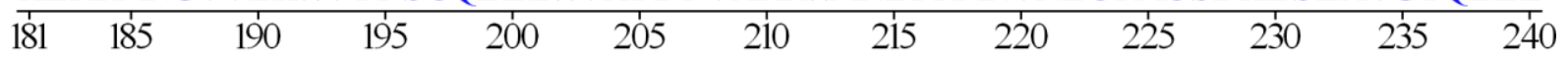
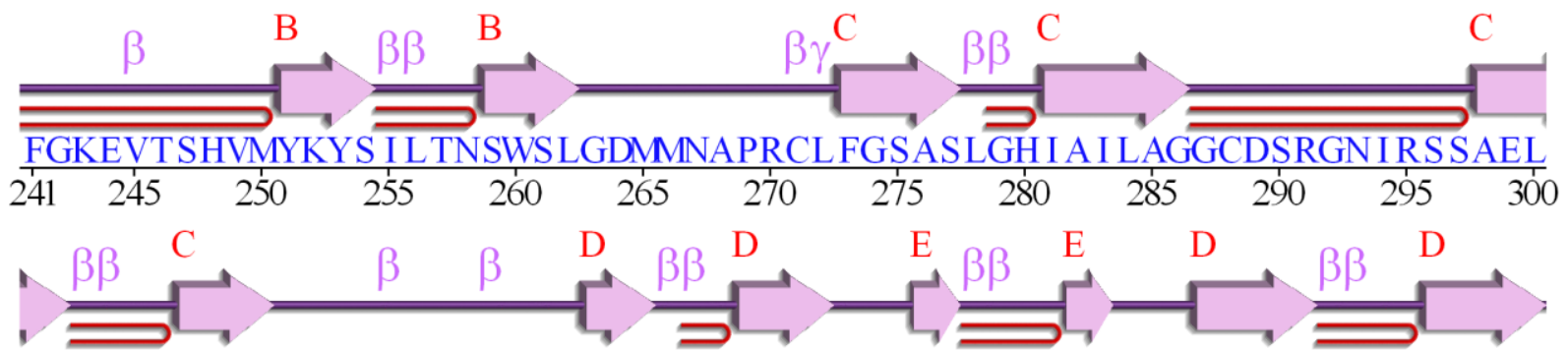

YDSEKETWEVLPDMI KPRKMCSGVFMDGKFCV I GG I GGSDSKLLTS SEEFDMETRTWKE I

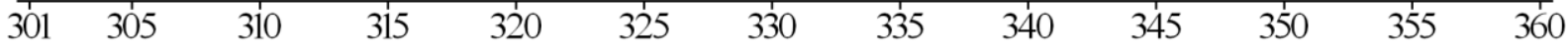

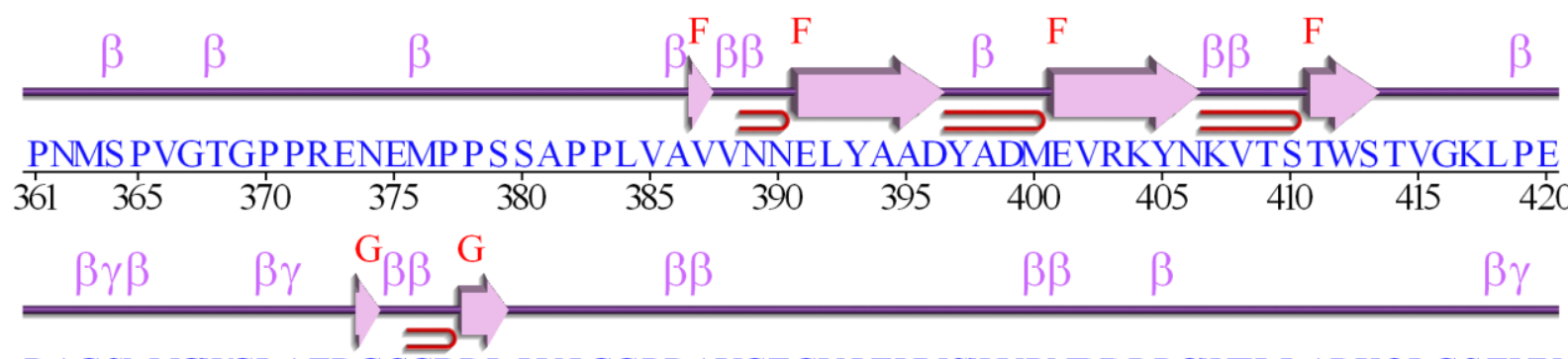

RAGSMNGWGLAFRGCGDRL I V I GGPRAYGEGV I EVNSWWPNDDP PQWTLLARKQLGS FVY

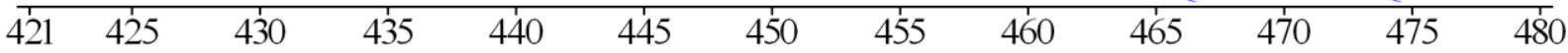

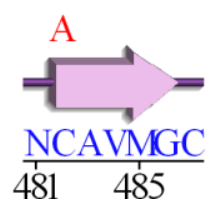

Key:

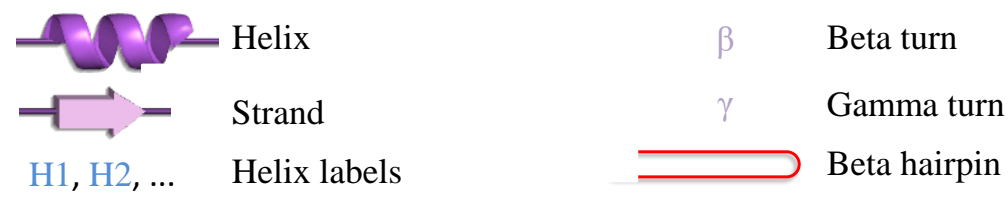

A, B, ... Strand by their sheet

Fig 5. Secondary structure of PmF-box 1 predicted by PDBsum. The amino acids sequence which involved in the formation of beta blade or beta sheet structures are in the red boxes. 
(a)

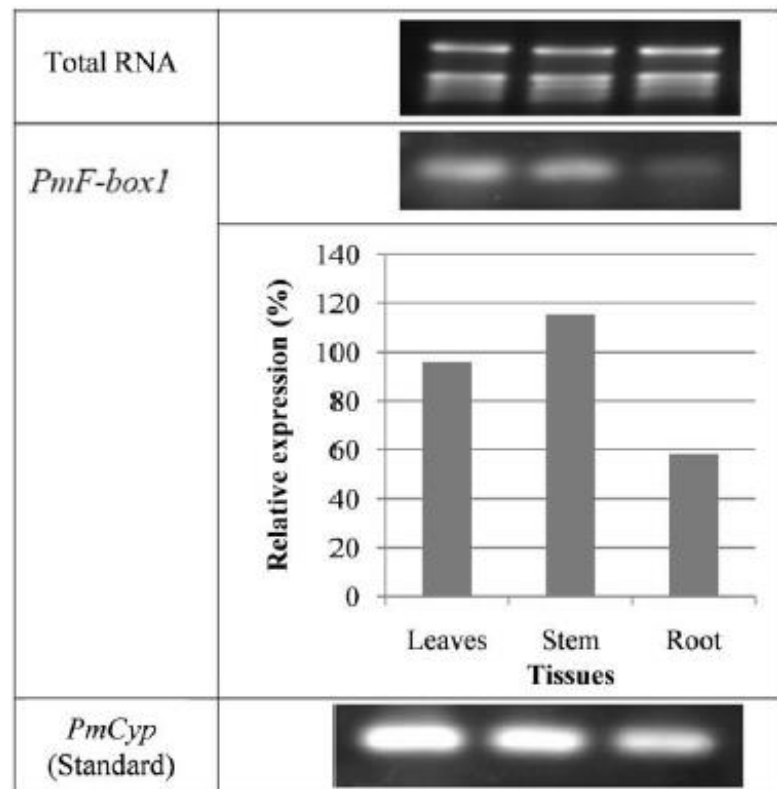

(b)

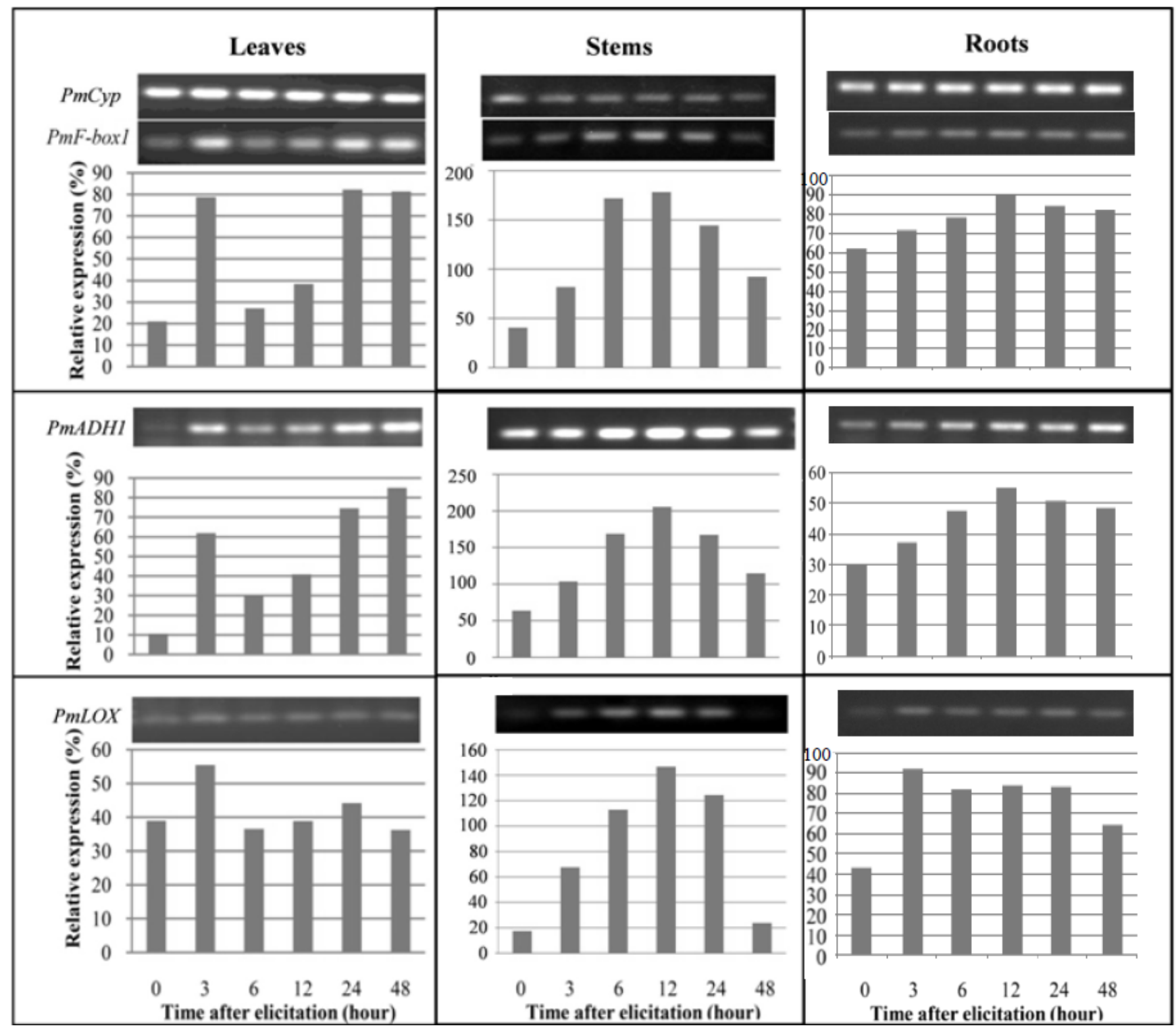


(c)
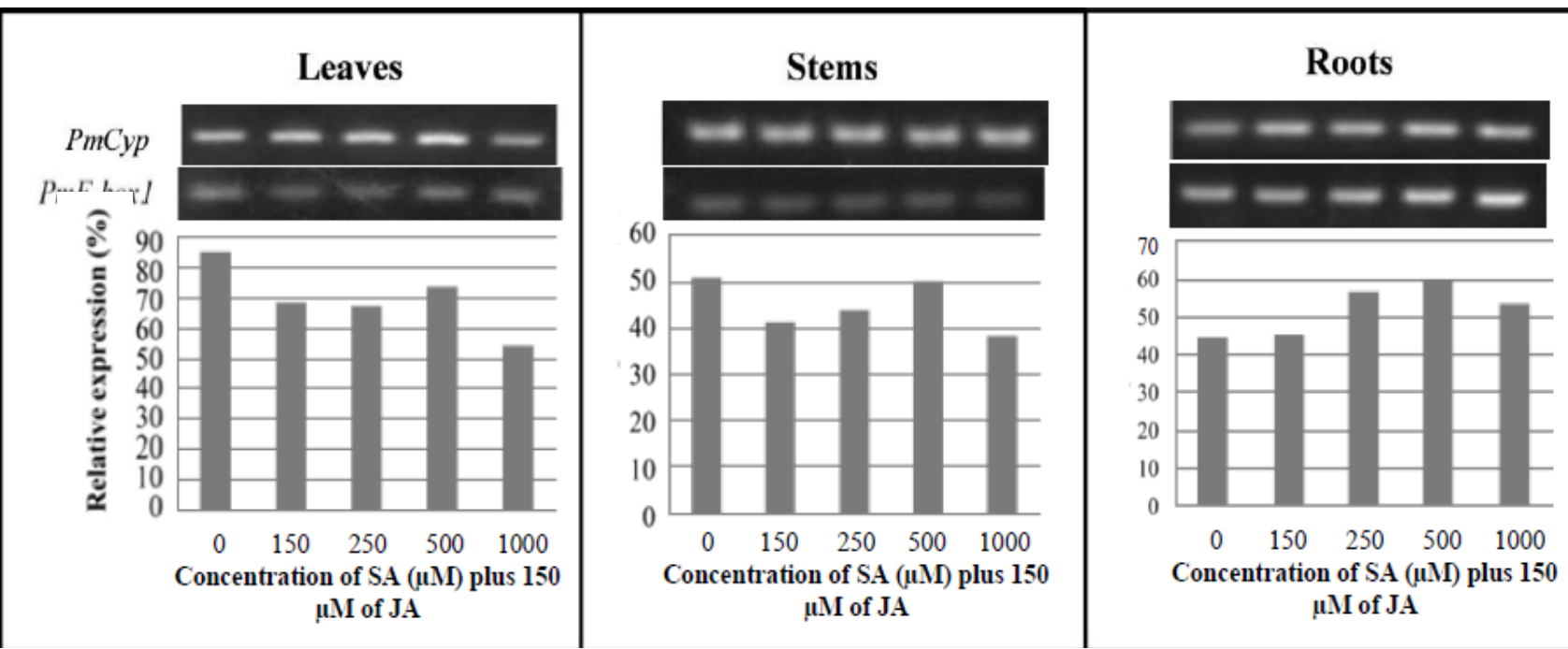

Fig 6. Expression patterns of PmF-box1, PmADH1 and PmLox. a) Expression analysis of PmF-box1 in different tissues at normal condition. b) Expression pattern of PmF-box1, PmADH1 and PmLOX in different tissues after elicited with $150 \mu \mathrm{M}$ JA. c) expression analysis of PmF-box1, PmADH1 and PmLOX after 12 hours elicitation with solution containing $150 \mu \mathrm{M}$ JA and SA with different concentrations.

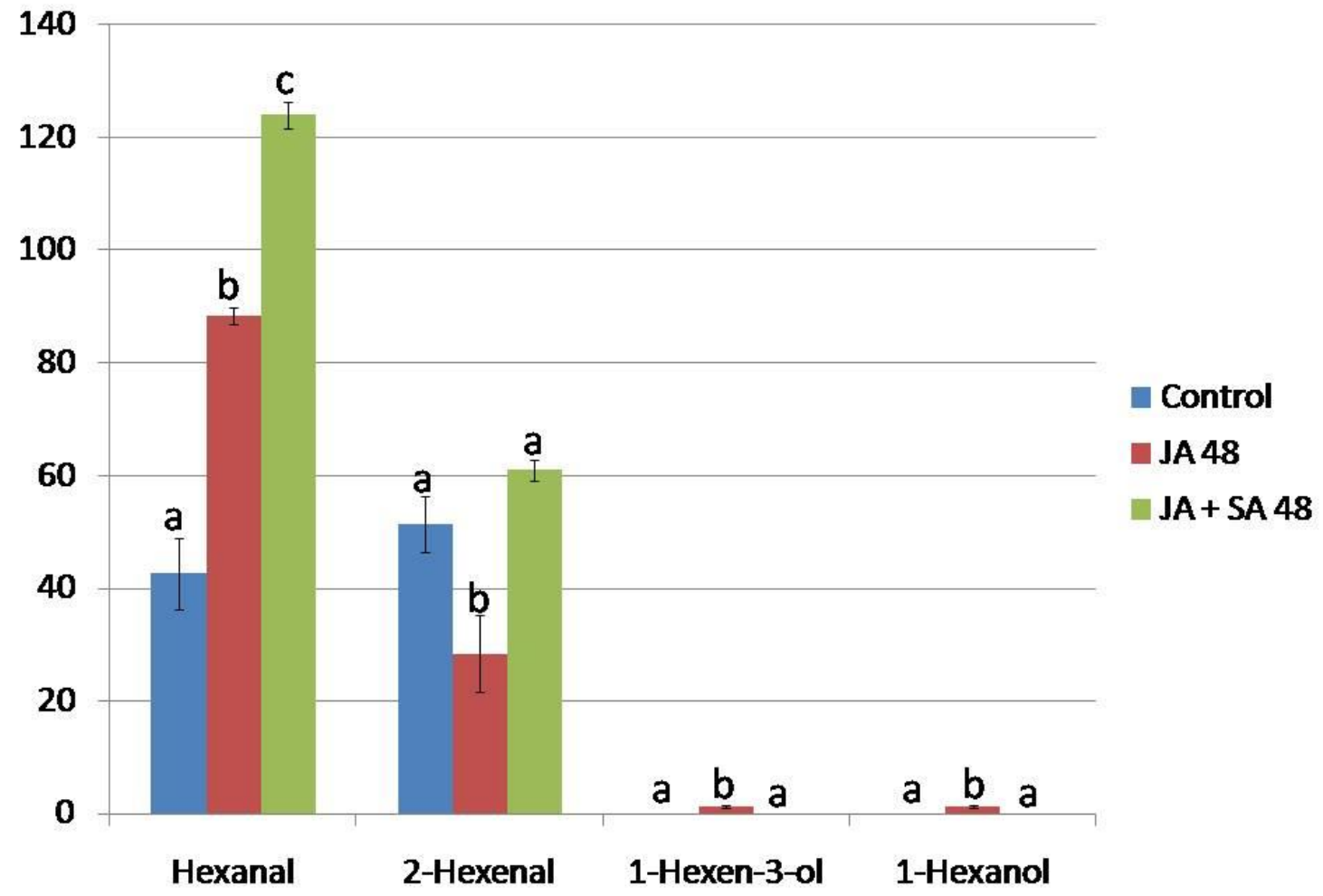

Fig 7. Production of GLV, 48 hours after treatment with $150 \mu \mathrm{M} \mathrm{JA}$ (JA 48h), and 48 hours after treatment with the combination of $150 \mu \mathrm{M} \mathrm{JA}$ and $150 \mu \mathrm{M}$ SA (JA+SA 48h). Values with different characters (a-c) are significantly different based on T-test $(\mathrm{P}<0.05$, $\mathrm{n}=3$ ). 
thaliana, Arabidopsis lyrata and Ricinus comunis. Multiple alignments of these F-box proteins have shown low similarities at the $\mathrm{N}$-terminal region and high similarities at the $\mathrm{C}$-terminal region, starting from $138^{\text {th }}$ residue of PmF-box 1 protein sequence (Fig 3).

The conserved motifs present on the protein sequence of PmF-box1 were identified through an online protein motif finding tool (myhits.isb-sib.ch/cgi-bin/motif_scan). . In addition to it, a multiple sequence alignment between PmF-box 1 and its protein homologs from other plant species was also generated to support the prediction of motif scan and to give a clearer view on the location of conserved amino acid residues in each identified motif. Based on the prediction of motif scan, two characteristic motifs were found in the PmF-box 1 protein sequence. The $\mathrm{N}$-terminal region of this protein possesses a conserved F-box motif (residues 146-189), whereas in the Cterminal region two conserved kelch motifs (residues 269-315 and 318-364) were identified (Fig 3). All identified motifs were located within the conserved region of PmF-box 1 . Thus, these results indicated that PmF-box1 belongs to the kelch repeat containing family of F-box proteins.

Based on the deduced amino acid sequence, the tertiary structure of PmF-box 1 was also predicted using I-Tasser, which is an online protein structure prediction tool. Fig $4 a \& b$ represent the front and the side view of the predicted PmF-box 1 protein structure. The F-box protein structure was coloured according to the identified domains and unidentified region as shown in the diagram of multiple sequence alignment (Fig 3). By observing the protein structure closely, it was shown that the C-terminal domain of PmF-box1 was forming the structure of $\beta$-propeller (flower-like structure) which consists of six $\beta$ stranded blades. The close-up version of $\mathrm{C}$-terminal domain was shown in the Fig $4 \mathrm{c}$ with the indication of conserved kelch repeat domains predicted by motif scan. In the diagram, some residues were coloured in red to indicate the conserved residues present on the protein structure which possess high structural similarity with other protein homologs. These conserved regions were predicted by I-Tasser and may be important for the stability of $P m$ F-box protein. In every blade, especially in conserved kelch repeat domain, there was a conserved tryptophan residue at the outermost strand. This residue is very important for the structural stability of PmF-box 1 by its participation in the formation of hydrogen bonding with tyrosine and arginine. In addition, glycine residues are also important as the backbone of beta propeller structure adopts a conformation that strongly favours glycine.

Even though there were only two kelch repeats identified through motif scan, which constituted two out of the six beta stranded blades (as shown in purple), there are four other beta sheets formed in this structure completing the structure of beta propeller (fower-like structure). The failure of sequence scanning algorithm to scan the other kelch repeats, which are present in PmF-box 1 protein is rather common as the amino acid residues in kelch repeat motif appeared to be less conserved as compared to its structure (Hudson \& Cooley 2000). In order to identify the regions of PmF-box 1 responsible for the formation of the remaining blade-shaped beta sheets, PDB sum (http://www.ebi.ac.uk/thornton-srv/databases/ pdbsum/Generate.html) was utilized to obtain the topology and secondary structure of the putative protein (Fig 5).

\section{Expression profiles of the PmF-box1 in Persicaria minor}

Previous studies indicated that the expression level of many Fbox genes were differentially regulated in distinct tissues of plant, therefore the level of PmF-boxl transcript in different tissues were also determined by semi-quantitative RT-PCR in the present study to investigate the spatial expression pattern of $P m F$-boxl. Under the normal conditions, expression of PmFboxl was highest in the stem and slightly lower in the leaves, but very low in the root samples as shown in the Fig 6a.

PmF-boxl was differentially expressed in different tissues after treatment with $150 \mu \mathrm{M}$ JA (Fig 6b). Expression of PmFboxl was found to be increased in the leaves, although it was not continuous. Expression pattern of PmF-boxl and two stress related genes, $P m L O X$ and $P m A D H 1$ were also compared. Expression pattern of PmF-boxl has shown high correlation with the expression pattern of $P m A D H 1(\mathrm{R}=0.90 \pm 0.05)$ and a moderate level of correlation with $P m L O X(\mathrm{R}=0.61 \pm 0.14)$.

\section{Production of green leaf voltiles (GLV)}

LOX is an important upstream enzyme of the oxylipin pathway and $\mathrm{ADH}$ is the oxylipin pathway downstream enzyme that involved in the inter-conversion of GLV aldehydes and alcohols. Effect of changes in the expression of these genes, on GLV production was determined by measuring the production of GLV during the period of maximum expression of PmLOX and PmADH1 in Persicaria minor and comparing the levels with control plants (without treatment). The level of expression of these genes, in the leaves of plants treated with JA and JA plus SA was found to be highest at 48 hours after the treatments. Four GLV compounds have been identified and measured, including hexanal, (E)-2-hexenal, 1-hexanol and 1hexen-3-ol. Fig 7 shows that hexanal production was significantly increased 48 hours after the treatment with 150 $\mu \mathrm{M} \mathrm{JA}$ and even higher after the treatment with $150 \mu \mathrm{M} J A$ plus $150 \mu \mathrm{M}$ SA. There was no significant change observed in the (E)-2-hexenal levels in all the treatments of this study. The contents of 1-hexanol and 1-hexen-2-ol were only detectable after 48 hours in the plants with JA treatment.

\section{Discussion}

In the present study, we obtained the full length cDNA sequence of PmF-boxl based on the expressed sequence tag (EST) and the deduced protein sequence was characterized. PmF-boxl has an ORF encoding a polypeptide of 487 amino acids and shares moderate sequence homology with other F-box proteins from other species. Among approximately 700 predicted F-box proteins encoded by the Arabidopsis genome, SKIP11 (NCBI database ID NP178390) has the highest homology with PmF-box1 protein sequence with the E value $7 \mathrm{e}^{-171}$. Unfortunately, information about SKIP11 is still limited as for now. Nevertheless, protein interaction analysis showed that SKIP11 was able to interact with ASK1 and ASK2 in Arabidopsis (Risseeuw et al. 2003). The putative PmF-box1 protein sequence contains an F-box motif in the $\mathrm{N}$-terminus and two Kelch repeat motifs in the C-terminus (Fig 3). These domains show high homology with other homologous F-box proteins from other plant species. From these data, we confirm that PmF-box 1 belongs to the Kelch repeat containing F-box (KFB) protein family.

$\mathrm{KFB}$ is one of the largest F-box family since there are approximately $100 \mathrm{KFB}$ genes that have been found in Arabidopsis thaliana (Schumann et al. 2011), and four of them have been functionally characterized; AFR, which involved in light signalling, and ZTL, FKF1 and LKP2, which are involved in the regulation of circadian oscillations and photomorphogenesis. ZTL, FKF1 and LKP2 have a LOV domain at the N-terminus of the F-box protein which acts as the light censoring domain for the F-box proteins (Fukamatsu et al. 2005; Sawa et al. 2007; Somers et al. 2004). Some other KFB proteins have been identified recently in chickpea (Cicer 
arietinum L.) (Jia et al. 2011), grape (Vitis vinifera L.) (Paquis et al. 2010) and mung bean (Phaseolus vulgaris L.) (Maldonado-Calderón et al. 2011), which have been shown to participate in the plant stress responses (Hassan et al. 2015b). Multiple sequences alignment analysis shows that the homology between PmF-box 1 protein sequences with all KFBtypes of F-box proteins mentioned above is very low. However, PmF-boxl is more likely to be involved in stress responses as the expression is increase after JA treatment and its protein lacks the LOV domain that is important in light signalling.

The kelch motif is an evolutionarily-widespread sequence motif of 44-56 amino acid residues, which was first identified in a Drosophila mutant (Xue and Cooley 1993). Previous studies have uncovered the consensus of the kelch motif that is characterized by four highly conserved residues comprising two adjacent glycines (G), and a pair of tyrosines ( $\mathrm{Y}$ ) and tryptophan (W) separated by about six residues (Prag and Adams 2003). There are two sequences in the PmF-box 1 found obeying this rule, so they are assigned as kelch repeats. A single kelch motif may forms a $\beta$ sheet structure which consists of four $\beta$ strands, and these $\beta$ sheets are arranged in a circle around a central axis to generate the $\beta$-propeller structure. This structure is important for protein-protein interaction between the F-box and the target protein (Schumann et al. 2011).

Analysis of the genomic sequence of PmF-boxl brings to identification of an intron located within the 5'untranslated region of the gene. The number and the distribution of introns in the F-box genes vary and rather specific for every type of encoded F-box protein (Gagne et al. 2002). So far, $40.76 \%$ of 687 genes in rice and $45 \%$ of 694 genes in Arabidopsis coding for F-box proteins identified are intron-less, whilst 18 out of 25 and almost all 100 genes encoding KFB-type protein in rice and Arabidopsis, respectively were found intron-less (Gagne et al. 2002; Jain et al. 2007). The number of intron in plant genes can be linked to the evolutionary process of the genes and may be either intron gain-dominated or intron loss-dominated (Roy and Penny 2007). Evolution of F-box genes can be classified as intron loss-dominated, because the loss of introns was associated to the recent evolutionary expansion of large F-box gen family (Yang et al. 2008). Based on these facts, we hypothesised that, genes encoding KFB-type proteins belong to the recently evolved genes while PmF-boxl gene belongs to the earlier genes encoding KFB-type proteins in plant.

In the previous study, the expression of PmF-boxl was found to be increased in Persicaria minor roots after JA treatment (Gor et al. 2010). Similar observations were also obtained for several other KFB proteins, such as chickpea (C. arietinum L.) grape (V. vinifera L.) and mung bean (P. vulgaris L.) (Jia et al. 2011; Maldonado-Calderón et al. 2011; Paquis et al. 2010). To study the expression profiles in more detail, we evaluated the expression of PmF-boxl in different tissues at different time points after treatment of the plants with JA and the combination of JA and SA. We also looked at the expression pattern of stress related genes $P m L O X$ and $P m A D H 1$ to see whether there was a correlation between the expressions of PmF-boxl with these genes.

The expressions of genes encoding KFB-type F-box protein, $\mathrm{ADH}$ and LOX are regulated by different regulatory mechanisms in Arabidopsis. Recently, some KFB-type F-box protein genes were found to be regulated by micro RNA from miR159 and miR2111 groups (Barrera-Figueroa et al. 2011; Patade and Suprasanna 2010). In A. thaliana, AtLOX is involved in JA signalling and is regulated by the transcription factor MYC2 and the repressor protein JAZ (Niu et al. 2011), whilst $A t A D H$ is involved in both the JA and ethylene signalling pathways and regulated by repressor- and activatortype Ethylene Response Factor (ERF) (Yang et al. 2011).
Interestingly, our data show that the correlation of expression profiles between PmF-boxl and PmADHI is very high, suggesting that there is relation between PmF-box 1 and the expression of PmADH1. One hypothesis which can be made is that PmF-box1 may positively regulate PmF-ADH1 through modulating ERF activities which is the immediate regulatory component for the expression of ADH1 genes in Arabidopsis.

Changes in the expression of PmLOX and PmADHI also influenced GLV production in Persicaria minor, especially hexanal and the six carbon alcohols. Hexanal was significantly increased 48 hours after treatment with JA alone and JA plus SA treatment at the time when expression of the genes was highest. The production of 1-hexanol and 1-hexen-2-ol also increased when they only became detectable after 48 hour treatment with JA. However, no significant increment was observed in the production of (E)-2-hexenal. It may be caused by the limited content of its precursor in the cell. Unlike 1hexanal, which is produced from linoleic acid, (E)-2-hexenal is produced from linolenic acid (Kenji 2006). Linolenic acid is the main constituent of several membranes in the plant cell. The content of linolenic acid is critical in influencing the fluidity and permeability of the membrane. Therefore, there is a maximum content of linolenic acid that the cell can bear so that it will not interfere with the cell function especially in photosynthetic cell (Domínguez et al. 2010).

SA is well known to have antagonistic effect on JA signaling pathway. However, our data shows that treatment with a combination of a solution containing equimolar $(150 \mu \mathrm{M}) \mathrm{JA}$ and SA induced the production of hexanal even higher from the leaves than the plants treated with JA alone. Furthermore, this observation is in accordance with the expression pattern of $P m L O X$, as it was also increased after 48 hours in the plants treated with JA plus SA compared to the plants treated with JA alone. This synergistic effect usually occurs when the concentration of SA used is considerably low as previously shown in maize (Engelberth et al. 2011) and tobacco (Mur et al. 2006). The mechanism behind this synergistic effect still remains elusive. In tobacco, the antagonistic effect of SA on the JA signaling pathway was visible only when the concentration of SA was higher than $250 \mu \mathrm{M}$ (Mur et al. 2006).

\section{Materials and Methods}

\section{Plant materials}

Persicaria minor plantlets were obtained from the Malacca Biotechnology Institute (Malacca, Malaysia). The plantlets were micro-propagated from the auxiliary buds by multiplenode stem cutting. The leaves were removed and the stems were cut into multiple-node segments. Each segment was then cultured in jam jars on Murashige and Skoog's (MS) (Duchefa, Netherlands) medium, supplemented with $30 \mathrm{~g} \mathrm{l}^{-1}$ sucrose (Hmbg, Germany) solidified with $0.3 \%$ gelrite (Duchefa, Netherlands) (Murashige and Skoog 1962). The pH of the MS medium was adjusted to 5.8. The node segments were grown in a growth chamber at $25 \pm 2^{\circ} \mathrm{C}$ with a photoperiod of $16 \mathrm{~h}$ of light and $8 \mathrm{~h}$ of darkness. The plantlets were sub-cultured for every 2 months.

\section{Extraction of total RNA}

Total RNA used for full length cDNA isolation and semiquantitative RT-PCR analysis was extracted from the leaves, stems and roots of $P$. minor following a previously published method (López-Gómez and Gómez-Lim 1992). The RNA integrity and quality were monitored by running $1.2 \%(\mathrm{w} / \mathrm{v})$ 
agarose formaldehyde gel electrophoresis and by NanoDrop spectrophotometer respectively.

\section{Cloning of PmF-box1 full-length cDNA by RACE}

The expressed sequence tag (EST) in the cDNA library was found to have a segment of a putative F-box gene and was used as template to amplify PmF-boxl by rapid amplification of 5' cDNA end and 3' cDNA end (RACE) using the SMARTer RACE cDNA Amplification Kit. Using $1 \mu \mathrm{g}$ of total RNA as template, full-length cDNA was generated following the manufacturer's instructions (Clontech, Mountain View, CA). All RACE PCRs were conducted using the Advantage 2 Polymerase (Clontech) following the manufacturer's instructions for SMARTer RACE.

All RACE PCR products were gel-extracted (QIAGEN Inc., Mississauga, ON) and cloned into pGEM-T easy vector (Promega, Madison, WI) at $4^{\circ} \mathrm{C}$ overnight using standard techniques. The recombinant plasmids were transformed, by heat shock method, into Sub-cloning Efficiency DH5a competent cells (Invitrogen), and plated on Luria broth (LB) agar supplemented with $50 \mathrm{mg} \mathrm{ml}^{-1}$ ampicillin. Individual colonies were grown overnight at $37^{\circ} \mathrm{C}$ in $\mathrm{LB}$ broth containing $50 \mathrm{mg} \mathrm{ml}-1$ ampicillin and the plasmid DNA was purified using the QIAprep Spin Miniprep Kit (QIAGEN Inc., Mississauga, $\mathrm{ON})$. In order to obtain the genomic sequence of PmF-box 1 gene, Persicaria minor genomic DNA was extracted from the leaves using the cetyltrimethylammonium bromide procedure (Doyle and Doyle 1987). The DNA sequence of the PmF-box1 gene was cloned using a pair of RACE validation primers listed in Table 1.

\section{Sequence analysis}

The full length sequence of PmF-boxl cDNA was used to deduce the amino acid sequence of the protein and the open reading frame $(\mathrm{ORF})$ of the gene. The sequence comparison was conducted through the database search using the BLASTp program (NCBI, National Center for Biotechnology Services, http://www.ncbi.nlm.nih.gov). Online software MyHits (http://myhits.isb-sib.ch/cgi-bin/motif_scan) was used for motif annotation in the deduced protein. PmF-box 1 amino acid sequence was aligned with F-box protein from other plant species using DNAMAN version 7 (Lynnon Biosoft, Vaudreuil, QC, Canada) with default parameters. The phylogenic tree was made using MEGA version 5.

\section{PmF-box1 protein structure prediction}

Structural model of putative PmF-box 1 was predicted using an online protein prediction tool I-Tasser (http://zhanglab.ccmb.med.umich.edu/I-TASSER). In I-Tasser, the 3-dimensional protein structural model is predicted based on the output of the multiple pairwise threading alignments through Local Meta-Threading-Server (LOMETS) and TASSER structure similarity simulation (Zhang 2008). The reference proteins that were used by I-Tasser to predict PmFbox 1 protein structure are shown in Table 2 .

\section{Spatial expression pattern of PmF-box1}

Total RNA was extracted from the leaves, stems and roots of Persicaria minor plants. Semi-quantitative reverse transcription PCR (RT-PCR) was performed for the expression analysis using the primers listed in the Table 3 . The intensity of the resulted bands corresponding to the targeted genes were normalised with the intensity of the bands corresponding to the reference gene encoding cyclophylin (PmCyp). The intensity of the bands were measured using ImageJ software and the results are presented as the relative expression of the targeted genes to the PmCyp expression signal. .

\section{Hormone treatment and expression profiling}

The plants were treated with JA and SA to analyze the expression patterns of PmF-boxl, PmLOX and PmADH1. For the treatment with JA and SA, aerial parts of the plants were sprayed with $5 \mathrm{~mL}$ of $150 \mu \mathrm{M}$ aqueous solution of the phytohormone. For the treatment involving a mixture of both phytohormones, the aerial parts of the plants were sprayed with $5 \mathrm{~mL}$ of an aqueous solution containing $150 \mu \mathrm{M}$ JA and 150 $\mu \mathrm{M}$ SA. Then the leaves were harvested, with a clean and sharp blade, after 3, 6, 12, 24, and 48 hours. Samples were also collected from the untreated plants to be used as control. The harvested leaves were immediately frozen in the liquid nitrogen and stored at $-80^{\circ} \mathrm{C}$ until used. Semi-quantitative reverse transcription PCR (RT-PCR) was performed for the gene expression analysis as described in previous section using the primers listed in the Table 2.

\section{Gas chromatography - mass spectrometry (GC-MS) analysis}

GC-MS analysis was carried out to compare the GLV content in the leaves at the lowest and the highest gene expression levels. Plants without elicitor treatment were used for the lowest expression. For JA (JA) and JA plus SA (JA+SA) treatments, the highest gene expression was shown at 48 hours after the treatment. Whereas, in the SA treated plants highest gene expression was observed at 72 hours after the treatment. The samples were prepared for the extraction of GLV using the solid phase micro extraction (SPME) technique. In this study, one gram of ground leaves material was taken in SPME vials; samples collected after $48 \mathrm{~h}$ for JA and JA+SA treated plants and after $72 \mathrm{~h}$ for SA treated plants. Then the volatiles emitted from the ground leaves in the vials were adsorbed onto a SPME fibre $(65 \mu \mathrm{m}$ Stable Flex PDMS/DVB, Supelco, PA, USA) for $30 \mathrm{~min}$ in a water bath maintained at $60^{\circ} \mathrm{C}$.

Analysis of the volatile components was initiated by injecting the SPME fibre into the injection port of a PerkinElmer Turbo Mass GC-MS machine (Shelton, CT, USA) for $5 \mathrm{~min}$ that was operated at $70 \mathrm{eV}$. A HP-5ms capillary column (Agilent Technologies, $30 \mathrm{~m} \times 0.25 \mathrm{~mm}$ id, $0.25 \mu \mathrm{m}$ film thickness) was used as stationary phase and helium gas was used as the carrier at a rate of $1.0 \mathrm{ml} \mathrm{min}^{-1}$. The GC oven temperature was set at $50^{\circ} \mathrm{C}$ and held for $3 \mathrm{~min}$, then increased by $10^{\circ} \mathrm{C} \mathrm{m^{-1 }}$ to $200^{\circ} \mathrm{C}$ and held for $3 \mathrm{~min}$ at this temperature. Finally, the temperature was increased to $300^{\circ} \mathrm{C}$ at a rate of $20^{\circ} \mathrm{C} \mathrm{min}$ mo $^{-1}$ and held for $4 \mathrm{~min}$ at this temperature. Both injector and detector temperatures were maintained at $250^{\circ} \mathrm{C}$. 6-undecanone was used as the internal standard and each analysis was performed in triplicate to assess the reproducibility of results.

\section{Conclusion}

In conclusion, a novel gene encoding a KFB-type F-box protein named PmF-boxl has been characterized from Persicaria minor that is probably involved in stress response through jasmonate signaling. The production of GLV in plants is considerably complex and may be influenced by many factors (Hassan et al. 2015a). Nevertheless, these data contribute to understanding more about the involvement of KFB-type F-box proteins in plant stress responses, especially in the production of GLV. From this study, we proposed that PmF-box1 may be involved in the GLV biosynthetic pathway, especially the 
alcohols through regulation of $P m A D H 1$ gene expression. With the successful expression of PmF-box 1 from E. coli cell, it would be interesting to investigate the protein structure and the possible target proteins of PmF-box1 in the future. It will contribute in understanding the function of KFB in plant physiology and specifically in the jasmonate signalling.

\section{Acknowledgments}

This study was financially supported by UKM grant. We would like to thank Prof Michael Meyrick Burrell and Dr Thelma Burrell for critical modification of the manuscript. The Research University (RU) Grant number is FRGS/1/2015/SG03/UKM/01/1.

\section{References}

Bari R, Jones JD (2009) Role of plant hormones in plant defence responses. Plant Mol Biol. 69(4):473-488.

Barrera-Figueroa B, Gao L, Diop N, Wu Z, Ehlers J, Roberts P, Close T, Zhu J-K, Liu R (2011) Identification and comparative analysis of drought-associated microRNAs in two cowpea genotypes. BMC Plant Biol. 11(1):127.

Chen G, Hackett R, Walker D, Taylor A, Lin Z, Grierson D (2004) Identification of a specific isoform of tomato lipoxygenase (TomloxC) involved in the generation of fatty acid-derived flavor compounds. Plant Physiol. 136(1):26412651.

Combet E, Henderson J, Eastwood DC, Burton KS (2006) Eight-carbon volatiles in mushrooms and fungi: properties, analysis, and biosynthesis. Mycoscience. 47(6):317-326.

Deng W, Grayburn WS, Hamilton-Kemp TR, Collins GB \& Hildebrand DF (1992) Expression of soybean-embryo lipoxygenase 2 in transgenic tobacco tissue. Planta. 187(2):203-208 .

Domínguez T, Hernández ML, Pennycooke JC, Jiménez P, Martínez-Rivas JM, Sanz C, Stockinger EJ, Sánchez-Serrano JJ, Sanmartín M (2010) Increasing Desaturase Expression in Tomato Results in Altered Aroma Profile and Enhanced Resistance to Cold Stress. Plant Physio. 153(2):655-665.

Doyle JJ, Doyle JL (1987) A rapid DNA isolation procedure for small quantities of fresh leaf tissue. Phytochemical Bulletin 19(1):11-15.

Engelberth J, Viswanathan S, Engelberth MJ (2011) Low concentrations of salicylic acid stimulate insect elicitor responses in Zea mays seedlings. J Chem Ecol. 37(3):263266.

Fukamatsu Y, Mitsui S, Yasuhara M, Tokioka Y, Ihara N, Fujita S, Kiyosue T (2005) Identification of LOV KELCH PROTEIN2 (LKP2)-interacting factors that can recruit LKP2 to nuclear bodies. Plant Cell Physiol. 46(8):1340-1349.

Gagne JM, Downes BP, Shiu S-H, Durski AM, Vierstra RD (2002) The F-box subunit of the SCF E3 complex is encoded by a diverse superfamily of genes in Arabidopsis. Proc Natl Acad Sci USA. 99(17):11519-11524 .

Gor M, Ismail I, Mustapha W, Zainal Z, Noor N, Othman R, Hussein Z (2010) Identification of cDNAs for jasmonic acidresponsive genes in Polygonum minus roots by suppression subtractive hybridization. Acta Physiol Planta. 33(2):283-294.

Gounaris Y (2010) Biotechnology for the production of essential oils, flavours and volatile isolates. A review. Flavour and Fragrance J. 25(5):367-386.

Hassan MN, Zainal Z, Ismail I (2015a) Green leaf volatiles: biosynthesis, biological functions and their applications in biotechnology. Plant Biotech J. 13(6):727-739.

Hassan MN, Zainal Z \& Ismail I (2015b) Plant kelch containing F-box proteins. RSC Adv. 5(53):42808-42814.
Heil M, Ton J (2008) Long-distance signalling in plant defence. Trends Plant Sci. 13(6):264-272.

Ho M, Ou C, Chan Yr, Chien CT, Pi H (2008) The utility F-box for protein destruction. Cell Mol Life Sci. 65(13):1977-2000.

Hua Z, Vierstra RD (2011) The Cullin-RING Ubiquitin-Protein Ligases. Ann Rev Plant Biol. 62(1):299-334.

Jain M, Nijhawan A, Arora R, Agarwal P, Ray S, Sharma P, Kapoor S, Tyagi AK, Khurana JP (2007) F-Box proteins in rice. Genome-wide analysis, classification, temporal and spatial gene expression during panicle and seed development, and regulation by light and abiotic Stress. Plant Physiol. 143(4):1467-1483.

Jia Y, Gu H, Wang X, Chen Q, Shi S, Zhang J, Ma L, Zhang H, Ma H (2011) Molecular cloning and characterization of an Fbox family gene CarF-box 1 from chickpea (Cicer arietinum L.). Mol Biol Rep. 1-9. doi:10.1007/s11033-011-0984-y

Kenji M (2006) Green leaf volatiles: hydroperoxide lyase pathway of oxylipin metabolism. Cur Opin Plant Biol. 9(3):274-280.

López-Gómez R, Gómez-Lim MA (1992) A Method for Extracting Intact RNA from Fruits Rich in Polysaccharides using Ripe Mango Mesocarp. HortSci. 27(5):440-442 .

León J, Royo Jn, Vancanneyt G, Sanz C, Silkowski H, Griffiths G, Sánchez-Serrano JJ (2002) Lipoxygenase H1 gene silencing reveals a specific role in supplying fatty acid hydroperoxides for aliphatic aldehyde production. J Biol Chem. 277(1):416-423.

Lechner E, Achard P, Vansiri Ar, Potuschak T \& Genschik P (2006) F-box proteins everywhere. Current Opinion in Plant Biology 9(6):631-638

Longhurst TJ, Tung HF, Brady CJ (1990) Developmental regulation of the expression of alcohol dehydrogenase in ripening tomato fruits. J Food Biochem. 14(6):421-433.

Maldonado-Calderón MT, Sepúlveda-García E, Rocha-Sosa M (2011) Characterization of novel F-box proteins in plants induced by biotic and abiotic stress. Plant Sci. 185186(0):208-217.

Mur LAJ, Kenton P, Atzorn R, Miersch O, Wasternack C (2006) The outcomes of concentration-specific interactions between salicylate and jasmonate signaling include synergy, antagonism, and oxidative stress leading to cell death. Plant Physiol. 140(1):249-262.

Murashige T, Skoog F (1962) A revised medium for rapid growth and bio assays with tobacco tissue cultures. Physiol Planta. 15(3):473-497.

Niu Y, Figueroa P, Browse J (2011) Characterization of JAZinteracting bHLH transcription factors that regulate jasmonate responses in Arabidopsis. J Exp Bot. 62(6):2143-2154.

Paquis S, Mazeyrat-Gourbeyre F, Fernandez O, Crouzet J, Clément C, Baillieul F, Dorey S (2010) Characterization of a F-box gene up-regulated by phytohormones and upon biotic and abiotic stresses in grapevine. Mol Biol Rep. 38(5):33273337.

Patade V, Suprasanna P (2010) Short-term salt and PEG stresses regulate expression of MicroRNA, miR159 in sugarcane leaves. J Crop Sci Biotechnol. 13(3):177-182.

Piesik D, Wenda-Piesik A, Kotwica K, Łyszczarz A, Delaney KJ (2011) Gastrophysa polygoni herbivory on Rumex confertus: Single leaf VOC induction and dose dependent herbivore attraction/repellence to individual compounds. J Plant Physiol. 168(17):2134-2138.

Prag S, Adams J (2003) Molecular phylogeny of the kelchrepeat superfamily reveals an expansion of $\mathrm{BTB} / \mathrm{kelch}$ proteins in animals. BMC Bioinformatics. 4(1):42

Risseeuw EP, Daskalchuk TE, Banks TW, Liu E, Cotelesage J, Hellmann H, Estelle M, Somers DE, Crosby WL (2003) 
Protein interaction analysis of SCF ubiquitin E3 ligase subunits from Arabidopsis. Plant J. 34(6):753-767.

Roy SW, Penny D (2007) Patterns of intron loss and gain in plants: Intron loss-dominated evolution and genome-wide comparison of $O$. sativa and A. thaliana. Mol Biol Evol. 24(1):171-181.

Sawa M, Nusinow DA, Kay SA, Imaizumi T (2007) FKF1 and GIGANTEA Complex Formation Is Required for Day-Length Measurement in Arabidopsis. Science. 318(5848):261-265.

Schumann N, Navarro-Quezada A, Ullrich K, Kuhl C, Quint M (2011) Molecular evolution and selection patterns of plant Fbox proteins with C-terminal kelch repeats. Plant Physiol. 155(2):835-850.

Somers DE, Fujiwara S (2009) Thinking outside the F-box: novel ligands for novel receptors. Trends Plant Sci. 14(4):206-213.

Somers DE, Kim W-Y, Geng R (2004) The F-Box protein ZEITLUPE confers dosage-dependent control on the circadian clock, photomorphogenesis, and flowering time. Plant Cell Online. 16(3):769-782.

Speirs J, Lee E, Holt K, Yong-Duk K, Steele Scott N, Loveys B, Schuch W (1998) Genetic manipulation of alcohol dehydrogenase levels in ripening tomato fruit affects the balance of some flavor aldehydes and alcohols. Plant Physiol. 117(3):1047-1058.

Tesniere C, Torregrosa L, Pradal M, Souquet J-M, Gilles C, Dos Santos K, Chatelet P, Gunata Z (2006) Effects of genetic manipulation of alcohol dehydrogenase levels on the response to stress and the synthesis of secondary metabolites in grapevine leaves. J Exp Bot. 57(1):91-99.
Thines B, Katsir L, Melotto M, Niu Y, Mandaokar A, Liu G, Nomura K, He SY, Howe GA, Browse J (2007) JAZ repressor proteins are targets of the SCFCOI1 complex during jasmonate signalling. Nature. 448(7154):661-665.

Verhage A, van Wees SC, Pieterse CM (2010) Plant immunity: it's the hormones talking, but what do they say? Plant Physiol. 154(2):536-540.

Wang C, Xing J, Chin C-K, Ho C-T, Martin CE (2001) Modification of fatty acids changes the flavor volatiles in tomato leaves. Phytochem. 58(2):227-232.

Wenda-Piesik A, Piesik D, Ligor T, Buszewski Ba (2010) Volatile organic compounds (VOCs) from cereal plants infested with crown rot: their identity and their capacity for inducing production of VOCs in uninfested plants. Int J Pest Manag. 56(4):377-383.

Xue F, Cooley L (1993) kelch encodes a component of intercellular bridges in Drosophila egg chambers. Cell. 72(5):681-693.

Yang CY, Hsu FC, Li JP, Wang NN, Shih MC (2011) The AP2/ERF transcription factor AtERF73/HRE1 modulates ethylene responses during hypoxia in Arabidopsis. Plant Physiol. 156(1):202-212.

Yang X, Kalluri UC, Jawdy S, Gunter LE, Yin T, Tschaplinski TJ, Weston DJ, Ranjan P, Tuskan GA (2008) The F-Box gene family is expanded in herbaceous annual plants relative to woody perennial plants. Plant Physiol. 148(3):1189-1200. 